\title{
Status and Prospects of the LHCb Experiment
}

\author{
Neville Harnew \\ University of Oxford, Oxford OX1 3RH, UK. \\ On behalf of the LHCb Collaboration
}

\begin{abstract}
The LHCb detector and trigger have undergone significant revision. A summary is given of the re-optimized LHCb detector and the status of its construction. The measurement of the unitarity triangle angle $\gamma$ in a number of complimentary channels is also reviewed.
\end{abstract}





\section{Introduction}

Prior to 2007, the first generation experiments to study CP-violation in the B-system, BaBar, Belle, CDF and D0, will measure the parameters of the unitarity triangle with varying degrees of precision.

- The quantity $\sin (2 \beta)$ will be well measured in the "gold plated" $\mathrm{B}_{\mathrm{d}}^{0} \rightarrow \mathrm{J} / \psi \mathrm{K}_{\mathrm{s}}^{0}$ channel, perhaps to a world precision exceeding $\sim 0.02$;

- The sides $\left|V_{t d} / V_{t s}\right|$ and $\left|V_{u b} / V_{c b}\right|$ will be known from $\mathrm{B}_{\mathrm{s}}^{0}-\overline{\mathrm{B}}_{\mathrm{s}}^{0}$ mixing and from $b \rightarrow u$ decays, respectively. These sides will be limited by theory to between $5-10 \%$;

- The quantity $\sin (2 \alpha)$ will be measured, but with poor statistical precision, maybe to $\sim 0.1$

- There will be no good measurement of the angle $\gamma$. The $\mathrm{B}_{\mathrm{s}}^{0}$ sector will be largely unexplored by the first generation experiments.

The LHC will enable a substantial improvement on the measurement of CP-violation parameters by providing an unprecedented source of B hadrons. The expected $b \bar{b}$ cross section of $500 \mu \mathrm{b}$ (which is to compared with the inelastic cross section of $\sim 80 \mathrm{mb}$ ), gives $10^{12} b \bar{b}$ pairs per year $\left(10^{7} \mathrm{~s}\right)$ at $14 \mathrm{TeV}$. Hence $\mathrm{LHCb}$ will make precision measurements of $\mathrm{CP}$ violation in many, often very rare, decay channels. Complementary measurements will be made of the same parameters in different channels, giving additional cross checks. In particular, $\mathrm{LHCb}$ will provide large statistics of $\mathrm{B}_{\mathrm{s}}^{0}$ mesons, allowing several redundant measurements to be made of the angle $\gamma$. This will be discussed in Section 3.

The correlation of the polar angles of $\mathrm{B}$ and $\overline{\mathrm{B}}$ hadrons, defined with respect to the LHC beam direction in the pp centre of mass system, is shown in Fig. 1. The LHCb experiment exploits the sharply peaked forward-backward $b \bar{b}$ production cross section. LHCb runs at a tuned LHC luminosity of $2 \times 10^{32} \mathrm{~cm}^{-2} \mathrm{~s}^{-1}$, which maximizes the number of single interactions per beam-crossing and keeps radiation damage to the detector at a tolerable level. Experimental attributes of $\mathrm{LHCb}$ include efficient $\pi / \mathrm{K}$ identification, excellent decay time resolution, and photon detection.

\section{Optimization of the $\mathrm{LHCb}$ Detector}

The LHCb detector is a single-arm dipole spectrometer, designed to maximize the efficiency for detecting forward-going $\mathrm{B}(\overline{\mathrm{B}})$ hadrons. A detailed description of the individual detector components and technologies can be found in the Technical Proposal (TP) [1]. The detector covers a forward angular aperture between approximately 10 and 250 mrad (300 mrad) in the non-bending (bending) plane. The detector will occupy Pit 8 of the LEP/LHC tunnel; DELPHI dismantling is now completed and the necessary modification work for the installation of $\mathrm{LHCb}$ has started. 
Recently there have been significant changes to the LHCb detector, in particular to the tracking system, the trigger and the upstream Ring Imaging Cherenkov (RICH) detector. The material budget of LHCb has increased significantly since the TP, following a realistic estimate of dead material and services. Hence the detector has been reoptimized, resulting in less material and an improved performance. The new layout of the LHCb experiment, the so-called "LHCb-Light" geometry, is shown in Fig. 2.

The detector consists of the following major components :

- The beampipe, which was aluminium at the time of the TP, but is now a beryllium/aluminium alloy. There is also the possibility for the first beampipe cone to be of all beryllium.

- A warm dipole magnet, which is centred at $z \sim 5 \mathrm{~m}$, has an aluminium conductor and gives a $\sim 4$ Tm field integral [2]. All contracts for the magnet have been signed, and production is well underway.

- The Vertex Locator (VELO) is installed inside the vacuum tank at the interaction region. There are 21 stations of single-sided silicon strip detectors which present $0.18 \mathrm{X}_{0}\left(0.04 \lambda_{0}\right)$, three stations less than in the TP. The sensors are $220 \mu \mathrm{m}$ thick and arranged into independent $r$ and $\phi$ planes [3], having $170 \mathrm{k}$ readout channels in total. The detector is an integral component of the Level-1 trigger, which dictates the choice of the $r-\phi$ geometry.

The VELO is crucial in all time-dependent CP violation studies. Good vertex and proper time resolution is essential in rejecting background, and in the study of $\mathrm{B}_{\mathrm{s}}^{0}-\overline{\mathrm{B}}_{\mathrm{s}}^{0}$ oscillations. Typical proper time resolutions are $45 \mathrm{fs}\left(\overline{\mathrm{B}}_{\mathrm{s}}^{0} \rightarrow \mathrm{D}_{\mathrm{s}}^{-} \pi^{+}\right)$and 30 fs $\left(\mathrm{B}_{\mathrm{s}}^{0} \rightarrow \mathrm{J} / \psi \phi\right)$.

The design of the VELO detector is progressing well, and the Technical Design Report (TDR) has been submitted [4].

- The Tracking System has undergone major revision since the TP. Nine tracking stations have been reduced to four and, as can be seen in Fig. 2, there are now no stations inside the magnet. The material budget of the tracking system has subsequently been reduced from $0.27 \mathrm{X}_{0}\left(0.11 \lambda_{0}\right)$ to $0.12 \mathrm{X}_{0}$.

The upstream station (TT) consists of $2 \times 2$ planes of silicon microstrips, currently undergoing final design. The downstream stations (T1-T3) have inner and outer regions which are segmented to maintain channel occupancies always below $10 \%$. Another major change from the TP is that the inner stations now use all-silicon microstrip technology, whereas the outer regions are equipped with $5 \mathrm{~mm}$ straw tubes [5]. A 3 m prototype LHCb straw tracker is currently operating in HERA-B.

The track reconstruction efficiency and the fraction of incorrectly reconstructed "ghost" tracks has been studied for the LHCb-Light geometry. Preliminary studies show that for a sample of $\mathrm{B}_{\mathrm{d}}^{0} \rightarrow \pi^{+} \pi^{-}$events and for momenta $>5 \mathrm{GeV} / \mathrm{c}$, the 
efficiency is typically better than $90 \%$, and the ghost-track fraction less than $5 \%$. Overall the reduction in the number of tracking stations has resulted in improved tracking performance due to fewer secondary interactions.

$\mathrm{LHCb}$ has an excellent mass resolution (eg. $\sim 17 \mathrm{MeV} / \mathrm{c}^{2}$ for the $\mathrm{B}$ mass in $\mathrm{B}_{\mathrm{d}}^{0} \rightarrow$ $\pi^{+} \pi^{-}$) which gives a powerful background suppression in the reconstruction of final states.

- Two RICH Detectors provide $2 \sigma \pi / \mathrm{K}$ separation over the momentum range 1$150 \mathrm{GeV} / \mathrm{c}(3 \sigma$ over the range $3-80 \mathrm{GeV} / \mathrm{c})$ [6]. RICH-1 is a combined $\mathrm{C}_{4} \mathrm{~F}_{10}$ gas and aerogel device sited upstream of the magnet, and $\mathrm{RICH}-2$ is a $\mathrm{CF}_{4}$ gas device sited downstream of the magnet. The RICH photodetector, a 1024-pixel LHC-speed Hybrid Photodiode (HPD), is currently under development. There will be a major review of the technology in 2003, with the Multianode Photomultiplier as the backup option.

The particle identification provided by the RICH detectors is crucial, firstly by reducing backgrounds in selected final states, and secondly by providing an efficient flavour tag (using kaons) of $\mathrm{B}$ or $\overline{\mathrm{B}}$. The power of the particle identification in reducing background in $\mathrm{B}_{\mathrm{d}}^{0} \rightarrow \pi^{+} \pi^{-}$events is demonstrated in Fig. 3, showing the signal before and after the particle identification criteria are applied. The purity and efficiency of the particle-identification selection are $84 \%$ and $90 \%$, respectively.

The RICH-1 arrangement has recently undergone a major re-design. RICH-1 now has composite mirrors with a mirror support system located outside the detector acceptance. The material budget of RICH-1 has subsequently been reduced from $0.14 \mathrm{X}_{0}\left(0.05 \lambda_{0}\right)$ to $0.085 \mathrm{X}_{0}$. In addition, the removal of shielding plates in front of the magnet gives $\sim 0.1 \mathrm{~T}$ of fringe field in the region of $\mathrm{RICH}-1$, which necessitates a new "vertical" RICH-1 layout with a two-mirror reflective geometry. To shield the photodetectors from the field, the HPDs of RICH-1 are surrounded by a 50 Tonne magnetic shielding box.

- Electromagnetic and Hadron Calorimeters provide electron, photon and hadron identification over most of the energy range and are crucial for triggering [7]. The ECAL is Shashlik type with a preshower, and has 5952 cells. The depth is $25 \mathrm{X}_{0}$ $(1.1 \lambda)$ and the resolution is $\sigma_{\mathrm{E}} / \mathrm{E}=0.10 / \sqrt{\mathrm{E}} \oplus 0.01$. Mass production of the ECAL has started and approximately $30 \%$ of modules have been produced [8]. The HCAL is a tile calorimeter with 1468 cells. The depth is $5.6 \lambda$ and the resolution is $\sigma_{\mathrm{E}} / \mathrm{E}=0.80 / \sqrt{\mathrm{E}} \oplus 0.1$. The first module of the HCAL has been built and tested, and mass production has now started [8].

- A Muon Detection System consists of five planes of multiwire proportional chambers (900 chambers in the inner and outer region) and multigap resistive plate chambers $^{1}$ (480 in the outer region) [9]. Four of these planes are embedded in iron

\footnotetext{
${ }^{1}$ The RPC technology has recently been dropped in favour of a full MWPC system
} 
absorbers. The system provides a high $\mathrm{p}_{\mathrm{T}}$ muon trigger as well as efficient muon identification. Production of all chambers is in preparation.

- A Four-Level Trigger System which is efficient, flexible and robust for B decays of interest [10]. It includes a Level-0 operating at an output rate of $\sim 1 \mathrm{MHz}$ which triggers on high $\mathrm{p}_{\mathrm{T}}$ muons, electrons and hadrons. Level-0 pre-selects high $\mathrm{p}_{\mathrm{T}}(>1-2 \mathrm{GeV} / \mathrm{c})$ muon tracks and calorimeter clusters. A Level-0 "pile-up" veto trigger ensures that $>80 \%$ of events will contain only one single beam interaction. The Level-1 trigger, formed from VELO tracks, then selects secondary vertices in software. The target output rate of Level-1 is $40 \mathrm{kHz}$, determined by the load on subsequent levels. The Level-2 trigger then runs fast algorithms on partial event data using the full detector resolution. At Level-3, complete event data are used to reconstruct the full B-decay chain, writing to mass storage at a target rate of $\sim 200 \mathrm{~Hz}$.

The Level-1 trigger has recently undergone major revision [10]:

- Level-0 information is now included in the Level-1 decision. For a given minimum bias output rate, this gives typically a factor 2 improvement in the efficiency for $\mathrm{B}_{\mathrm{d}}^{0} \rightarrow \pi^{+} \pi^{-}$events.

- The fringe field in the region between the VELO and the magnet results in $\sim 0.4 \mathrm{~T}$ at the first tracking station, TT. Studies have shown that the momentum resolution achievable at Level-1 using tracks from the VELO and TT is $\sigma(1 / \mathrm{p}) \approx 0.2 / \mathrm{p} \oplus 0.01(\mathrm{p}$ in $\mathrm{GeV} / \mathrm{c})$. This allows a more efficient rejection of fake vertices which result from secondary interactions.

Preliminary studies show that at a $40 \mathrm{kHz}$ output rate, the new Level-1 design gives an efficiency for $\mathrm{B}_{\mathrm{d}}^{0} \rightarrow \pi^{+} \pi^{-}$events better than $85 \%$. The combined efficiency of all trigger levels is typically $30 \%$ for reconstructable events within the spectrometer.

\section{Measurements of the angle $\gamma$}

A major strength of the LHC is that it provides a copious number of $\mathrm{B}_{\mathrm{s}}^{0}$ mesons. This allows the angle $\gamma$ to be measured by LHCb in a variety of complementary ways, examples of which are described below. Note that all the performance figures quoted in this section relate to the LHCb TP geometry, prior to detector optimization.

\section{$3.1 \quad \mathrm{~B}_{\mathrm{s}}^{0} \rightarrow \mathrm{D}_{\mathrm{s}}^{-} \mathrm{K}^{+}$}

The channel $\mathrm{B}_{\mathrm{s}}^{0} \rightarrow \mathrm{D}_{\mathrm{s}}^{-} \mathrm{K}^{+}$and its charge conjugate states provide a theoretically clean measurement of the angle $(\gamma-2 \delta \gamma)$ [11]. Four time-dependent decay rates are measured, $\mathrm{B}_{\mathrm{s}}^{0} \rightarrow \mathrm{D}_{\mathrm{s}}^{-} \mathrm{K}^{+}, \mathrm{B}_{\mathrm{s}}^{0} \rightarrow \mathrm{D}_{\mathrm{s}}^{+} \mathrm{K}^{-}, \overline{\mathrm{B}}_{\mathrm{s}}^{0} \rightarrow \mathrm{D}_{\mathrm{s}}^{-} \mathrm{K}^{+}$and $\overline{\mathrm{B}}_{\mathrm{s}}^{0} \rightarrow \mathrm{D}_{\mathrm{s}}^{+} \mathrm{K}^{-}$, which yield values for $(\gamma-2 \delta \gamma)$ and the strong phase difference, $\Delta$. The importance of particle identification 
and good mass resolution is demonstrated in Fig. 4. In the absence of particle identification, the signal is dominated by background from $\mathrm{B}_{\mathrm{s}}^{0} \rightarrow \mathrm{D}_{\mathrm{s}}^{-} \pi^{+}$decays (ie. the $\mathrm{B}_{\mathrm{s}}^{0}$ mixing channel, which has a branching fraction 10 times higher than the signal channel).

Sensitivities per year to $(\gamma-2 \delta \gamma)$ depend on $(\gamma-2 \delta \gamma), \Delta$ and the $\mathrm{B}_{\mathrm{s}}^{0}$-mixing parameter $x_{\mathrm{s}}$. LHCb will record $\sim 2400$ events per one year of running, giving a sensitivity of $3^{\circ}-16^{\circ}$ in $(\gamma-2 \delta \gamma)$.

\section{$3.2 \quad \mathrm{~B}_{\mathrm{d}}^{0} \rightarrow \pi^{+} \pi^{-}$and $\mathrm{B}_{\mathrm{s}}^{0} \rightarrow \mathrm{K}^{+} \mathrm{K}^{-}$}

The channels $\mathrm{B}_{\mathrm{d}}^{0} \rightarrow \pi^{+} \pi^{-}$and $\mathrm{B}_{\mathrm{s}}^{0} \rightarrow \mathrm{K}^{+} \mathrm{K}^{-}$provide a measurement of the angle $\gamma$. The time-dependent decay asymmetries $\mathrm{A}_{\mathrm{CP}}=\mathrm{A}_{\mathrm{dir}} \cos \Delta_{\mathrm{m}} \mathrm{t}+\mathrm{A}_{\mathrm{mix}} \sin \Delta_{\mathrm{m}} \mathrm{t}$, where $\Delta_{\mathrm{m}}$ is the mass difference between the mass eigenstates, are measured in both channels [12]. The $\pi \pi$ and KK coefficients are fitted from the data, and related by U-spin symmetry to extract $\gamma$. Particle identification and good mass resolution is vital for this measurement.

Approximately 5000 events are expected per year in each channel. The angle $\gamma$ can be measured to a precision of $5^{\circ}-10^{\circ}$ after one year of running, limited by theoretical uncertainty.

\section{$3.3 \quad \mathrm{~B}_{\mathrm{d}}^{0} \rightarrow \mathrm{D}^{\star} \pi$ and $\mathrm{D}^{\star} 3 \pi$}

The channels $\mathrm{B}_{\mathrm{d}}^{0} \rightarrow \mathrm{D}^{\star \pm} \pi^{\mp}$ and $\mathrm{D}^{\star} 3 \pi$ provide measurements of the angle $(2 \beta+\gamma)$ via four time-dependent decay rates $[1,13]$. Small CP asymmetries, $\sim 1 \%$, result from the interference of the tree diagram with the doubly-Cabibbo suppressed decay into the same final state. The LHCb sensitivity of these channels has been studied. The method involves an inclusive $\mathrm{D}^{\star}$ reconstruction technique which yields $\sim 500 \mathrm{k}$ events per year in the channel $\mathrm{B}_{\mathrm{d}}^{0} \rightarrow \mathrm{D}^{\star \pm} \pi^{\mp}$, with a signal to background of $\sim 5$. Including the $\mathrm{D}^{\star} \mathrm{a}_{1}\left(\mathrm{a}_{1} \rightarrow 3 \pi\right)$ channel gives an additional $360 \mathrm{k}$ events/year. An efficient hadron trigger is vital to provide the large statistics required.

The error on $(2 \beta+\gamma)$ is shown as a function of $(2 \beta+\gamma)$ in Fig. 5, which assumes no strong phase difference. In this case the angle $\gamma$ can be measured to a precision better than $5^{\circ}$ after five years of running, assuming $\beta$ is measured from $\mathrm{B}_{\mathrm{d}}^{0} \rightarrow \mathrm{J} / \psi \mathrm{K}_{\mathrm{s}}^{0}$.

\section{$3.4 \quad \mathrm{~B}_{\mathrm{d}}^{0} \rightarrow \overline{\mathrm{D}}^{0} \mathrm{~K}^{\star 0}$}

The channel $\mathrm{B}_{\mathrm{d}}^{0} \rightarrow \overline{\mathrm{D}}^{0} \mathrm{~K}^{\star 0}$ and its charge conjugate states provide a determination of the angle $\gamma$ via a measurement of relative branching ratios [14]. Six time-integrated decay rates are measured : $\mathrm{B}_{\mathrm{d}}^{0} \rightarrow \mathrm{D}^{0} \mathrm{~K}^{\star 0}, \mathrm{~B}_{\mathrm{d}}^{0} \rightarrow \overline{\mathrm{D}}^{0} \mathrm{~K}^{\star 0}, \mathrm{~B}_{\mathrm{d}}^{0} \rightarrow \mathrm{D}_{\mathrm{CP}=+1}^{0} \mathrm{~K}^{\star 0}, \overline{\mathrm{B}}_{\mathrm{d}}^{0} \rightarrow \overline{\mathrm{D}}^{0} \overline{\mathrm{K}}^{\star 0}$, $\overline{\mathrm{B}}_{\mathrm{d}}^{0} \rightarrow \mathrm{D}^{0} \overline{\mathrm{K}}^{\star^{0}}$ and $\overline{\mathrm{B}}_{\mathrm{d}}^{0} \rightarrow \mathrm{D}_{\mathrm{CP}=+1}^{0} \overline{\mathrm{K}}^{\star^{0}}$. $\mathrm{D}_{\mathrm{CP}=+1}^{0}$ signifies the decay of the $\mathrm{D}^{0}$ into a $\mathrm{CP}$ eigenstate, i.e. $\mathrm{K}^{+} \mathrm{K}^{-}$or $\pi^{+} \pi^{-}$, otherwise $\mathrm{D}^{0} \rightarrow \mathrm{K}^{+} \pi^{-}$decays are considered. Since visible branching ratios are very small $\left(10^{-8}-10^{-7}\right)$, the measurements are only possible with a high-rate forward detector utilizing particle identification.

The LHCb sensitivity in these channels to $\gamma$ has been evaluated. Small branching fractions result in an annual yield of only 1700 events in all six channels, hence the 
method is statistics limited. The $\mathrm{B}_{\mathrm{d}}^{0}$ reconstructed in the $\mathrm{B}_{\mathrm{d}}^{0} \rightarrow \overline{\mathrm{D}}^{0} \mathrm{~K}^{\star 0}$ signal is shown in Fig. 6, demonstrating the importance of particle identification. Using this method, $\mathrm{LHCb}$ can measure the angle $\gamma$ to a precision of $\sim 10^{\circ}$ after one year of running.

\section{Performance Summary}

The physics performance of LHCb after one year's running (taken to be $10^{7} \mathrm{~s}$ ) is summarized in Table 1. This shows event yields of reconstructable and tagged events for a luminosity of $1.5 \times 10^{32} \mathrm{~cm}^{-2} \mathrm{~s}^{-1}$. Tagging efficiencies and wrong tag fractions are typically $40 \%$ and $30 \%$, respectively. The performance figures are currently being re-evaluated for the new LHCb-Light geometry and the improved trigger.

In summary, $\mathrm{LHCb}$ will perform a study of $\mathrm{CP}$ violation with unprecedented precision in many different and complimentary channels, and will provide a sensitive test of the Standard Model and physics beyond. The detector provides good particle identification, vertexing, and has an efficient and flexible trigger. Construction of the detector has started and is progressing well. The $\mathrm{LHCb}$ experiment will be ready for data-taking in 2007.

\section{Acknowledgments}

I would like to thank colleagues in the LHCb Collaboration who have provided invaluable assistance, in particular O. Callot, R. Forty, T. Ketel, F. Teubert, T. Nakada and G. Wilkinson. I also thank S. Erhan, P. Schlein and the Beauty2002 local committee, in particular B. Adeva, for making the conference in Santiago de Compostella so enjoyable. 


\section{References}

[1] "A Large Hadron Collider Beauty Experiment for Precision Measurements of CP Violation and Rare Decays"; LHCb Technical Proposal, LHCC 98/04, LHCC/P4 (Feb. 1998).

[2] LHCb Magnet Technical Design Report. CERN/LHCC 2000-007.

[3] U. Parzefall. "LHCb Silicon: VELO and Inner Tracker." These proceedings.

[4] VELO Technical Design Report. CERN/LHCC 2001-011.

[5] LHCb Outer Tracker Technical Design Report. CERN/LHCC 2001-024.

[6] LHCb RICH Technical Design Report. CERN/LHCC 2000-037.

[7] LHCb Calorimeters Technical Design Report. CERN/LHCC 2000-036.

[8] A. Goloutvin. "LHCb Calorimetry System." These proceedings.

[9] LHCb Muon System Technical Design Report. CERN/LHCC 2001-010.

[10] H. Dijkstra. "The LHCb Trigger." These proceedings.

[11] See for example R. Aleksan, I. Dunietz and B. Kayser, Z. Phys. C54 653 (1992).

[12] R. Fleischer. CERN-TH/2000-101

[13] I. Dunietz, Beauty'97, Nucl. Instr. Meth. A408 (1998) 14.

[14] M. Gronau \& D. Wyler, Phys. Lett. B265 (1991) 172. 


\begin{tabular}{|l|l|l|l|l|}
\hline Measurement & Channel & $\begin{array}{l}\text { Trigger } \\
\text { Efficiency }\end{array}$ & $\begin{array}{l}\text { Event } \\
\text { Yield }\end{array}$ & $\begin{array}{l}\text { Sensitivity } \\
1 \text { year }\end{array}$ \\
\hline $\sin (2 \beta)$ & $\mathrm{B}_{\mathrm{d}}^{0} \rightarrow \mathrm{J} / \psi \mathrm{K}_{\mathrm{s}}^{0}$ & $36 \%$ & $45.6 \mathrm{k}$ & $0.3^{\circ}-0.5^{\circ}$ \\
$\sin (2 \alpha)$ & $\mathrm{B}_{\mathrm{d}}^{0} \rightarrow \pi^{+} \pi^{-}$ & $30 \%$ & $6.9 \mathrm{k}$ & $2^{\circ}-10^{\circ}$ \\
$\sin (2 \alpha), \cos (2 \alpha)$ & $\mathrm{B}_{\mathrm{d}}^{0} \rightarrow \rho \pi$ & $20 \%$ & $1.3 \mathrm{k}$ & $5^{\circ}-15^{\circ}$ \\
$2 \beta+\gamma$ & $\mathrm{B}_{\mathrm{d}}^{0} \rightarrow \mathrm{D}^{\star} \pi, 3 \pi$ & $33 \%$ & $860 \mathrm{k}$ & down to $7^{\circ}$ \\
$\gamma-2 \delta \gamma$ & $\mathrm{B}_{\mathrm{s}}^{0} \rightarrow \mathrm{D}_{\mathrm{s}} \mathrm{K}$ & $28 \%$ & $2.4 \mathrm{k}$ & $3^{\circ}-16^{\circ}$ \\
$\gamma$ & $\mathrm{B}_{\mathrm{d}}^{0} \rightarrow \overline{\mathrm{D}}^{0} \mathrm{~K}^{\star 0}$ & $21 \%$ & $0.4 \mathrm{k}$ & $4^{\circ}-18^{\circ}$ \\
$\delta \gamma$ & $\mathrm{B}_{\mathrm{s}}^{0} \rightarrow \mathrm{J} / \psi \phi$ & $38 \%$ & $44 \mathrm{k}$ & $0.6^{\circ}$ \\
$x_{s}$ & $\mathrm{~B}_{\mathrm{s}}^{0} \rightarrow \mathrm{D}_{\mathrm{s}}^{-} \pi^{+}$ & $28 \%$ & $34.5 \mathrm{k}$ & $<90$ \\
Rare decay & $\mathrm{B}_{\mathrm{s}}^{0} \rightarrow \mu^{+} \mu^{-}$ & $95 \%$ & 10 & $4.4 \sigma \mathrm{S} . \mathrm{M}$. \\
Rare decay & $\mathrm{B}_{\mathrm{d}}^{0} \rightarrow \mathrm{K}^{\star 0} \gamma$ & $8 \%$ & $26 \mathrm{k}$ & - \\
& & & \\
\hline
\end{tabular}

Table 1: Performance summary of LHCb in a selection of benchmark channels for one year of operation at a luminosity of $1.5 \times 10^{32} \mathrm{~cm}^{-2} \mathrm{~s}^{-1}$. The quoted numbers are the errors on the parameter in question, unless specified otherwise. The $\alpha$ measurement in the $\mathrm{B}_{\mathrm{d}}^{0} \rightarrow \pi^{+} \pi^{-}$channel assumes no penguin contribution. The $\mathrm{B}_{\mathrm{s}}^{0} \rightarrow \mu^{+} \mu^{-}$channel assumes a Standard Model branching ratio of $3.5 \times 10^{-9}$. 


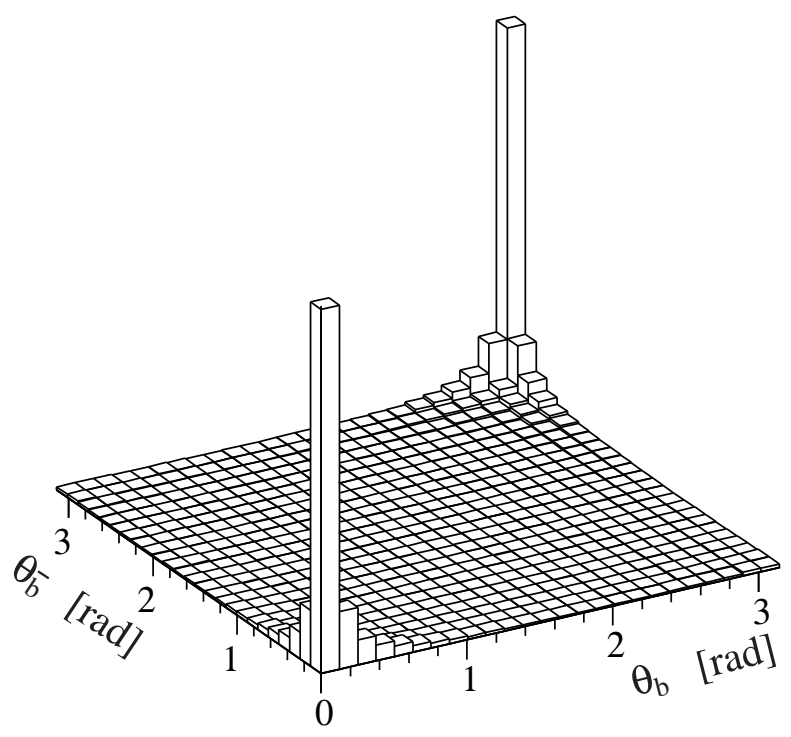

Figure 1: The distribution (arbitrary scale) of polar angles of hadrons containing $b$ and $\bar{b}$ quarks at the LHC. 


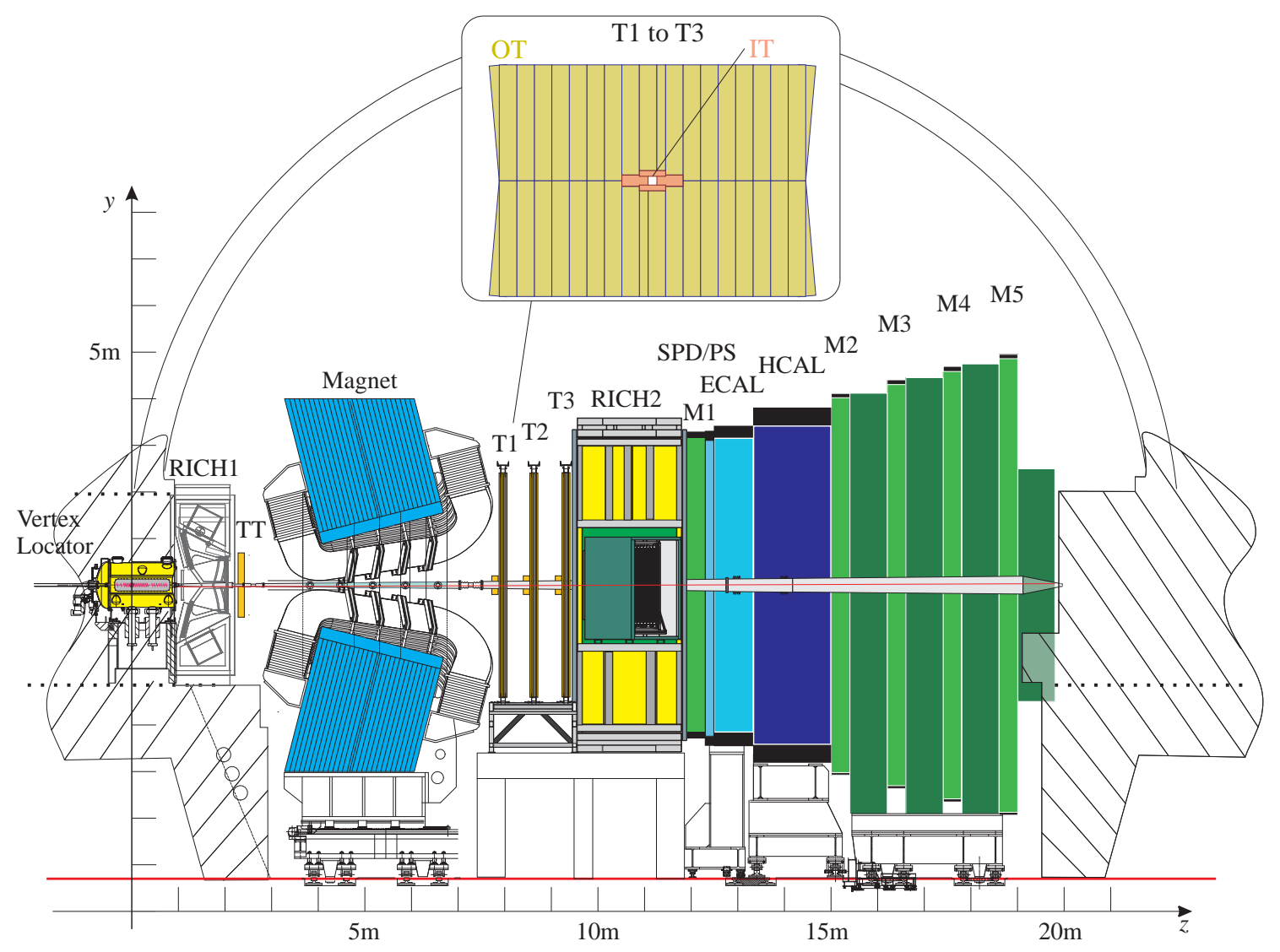

Figure 2: The optimized "LHCb-Light" spectrometer. The layout of the inner and outer regions of the T1-T3 chambers is highlighted. 

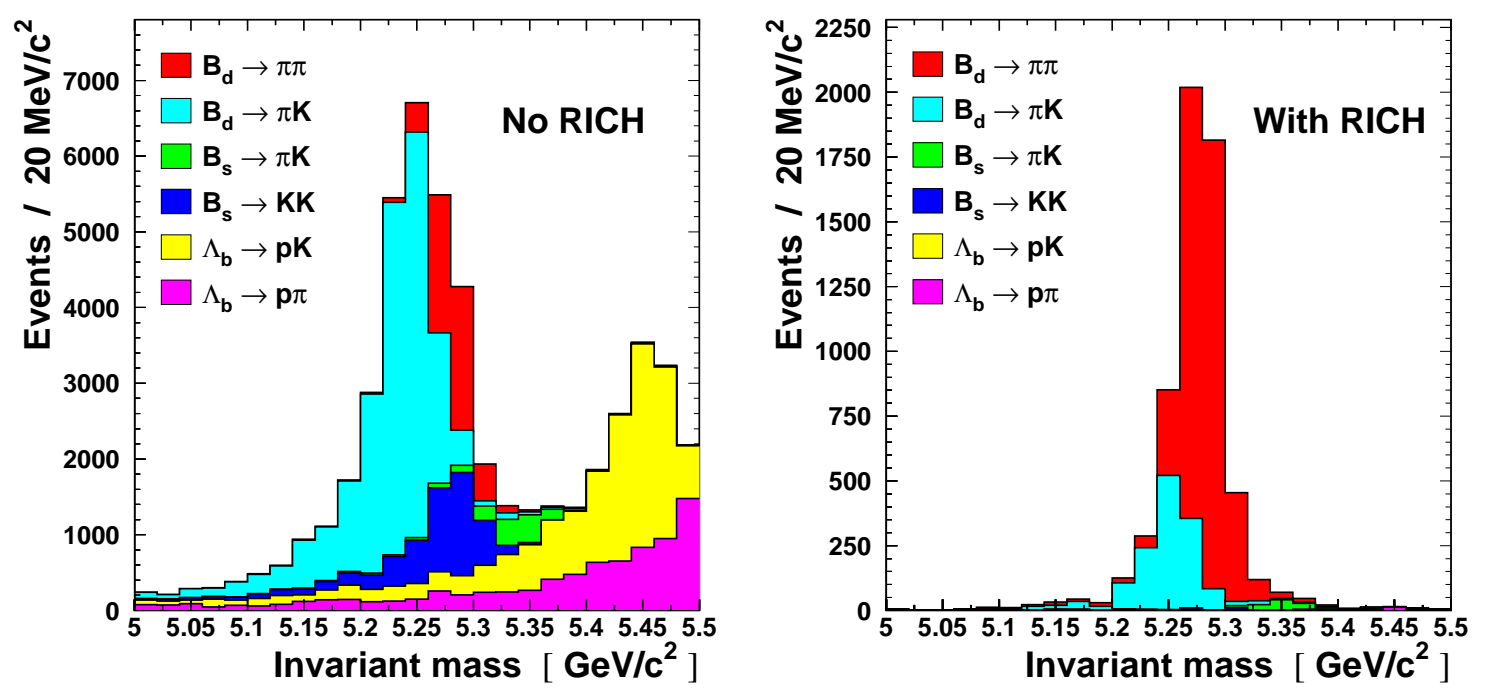

Figure 3: $\mathrm{B}_{\mathrm{s}}^{0}$ mass peak from $\pi^{+} \pi^{-}$combinations, showing the background from other $\mathrm{B}$ decay channels, without and with RICH information.
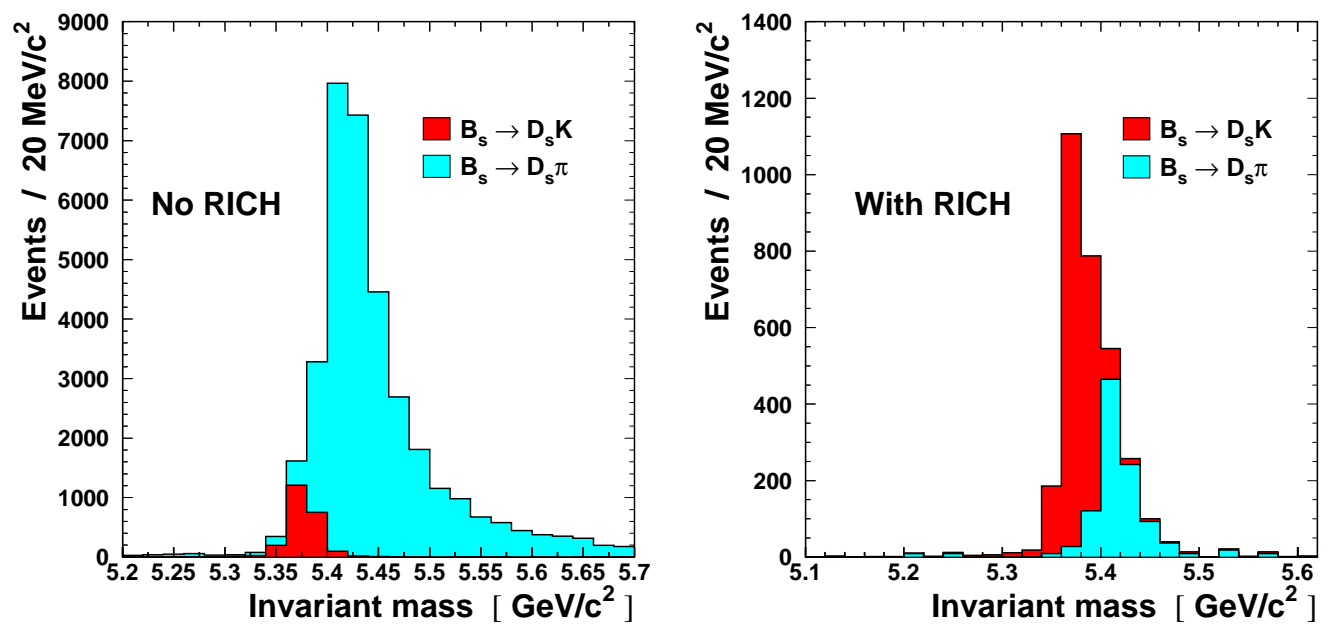

Figure 4: The $\mathrm{B}_{\mathrm{s}}^{0}$ mass peak from $\mathrm{D}_{\mathrm{s}}^{-} \mathrm{K}^{+}$combinations, showing the background from $\mathrm{D}_{\mathrm{s}}^{-} \pi^{+}$, with and without RICH information. 


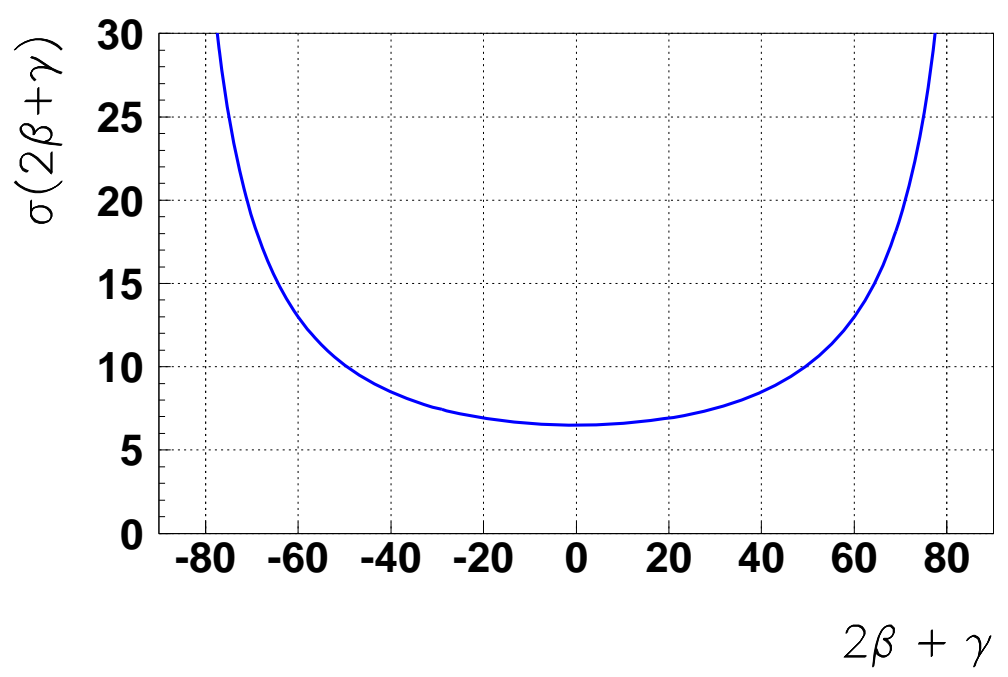

Figure 5: The error on $(2 \beta+\gamma)$ as a function of $(2 \beta+\gamma)$ after one year of LHCb running. The curve assumes no strong phase difference and a perfect knowledge of the ratio of the tree and Cabibbo-suppressed decay amplitudes.

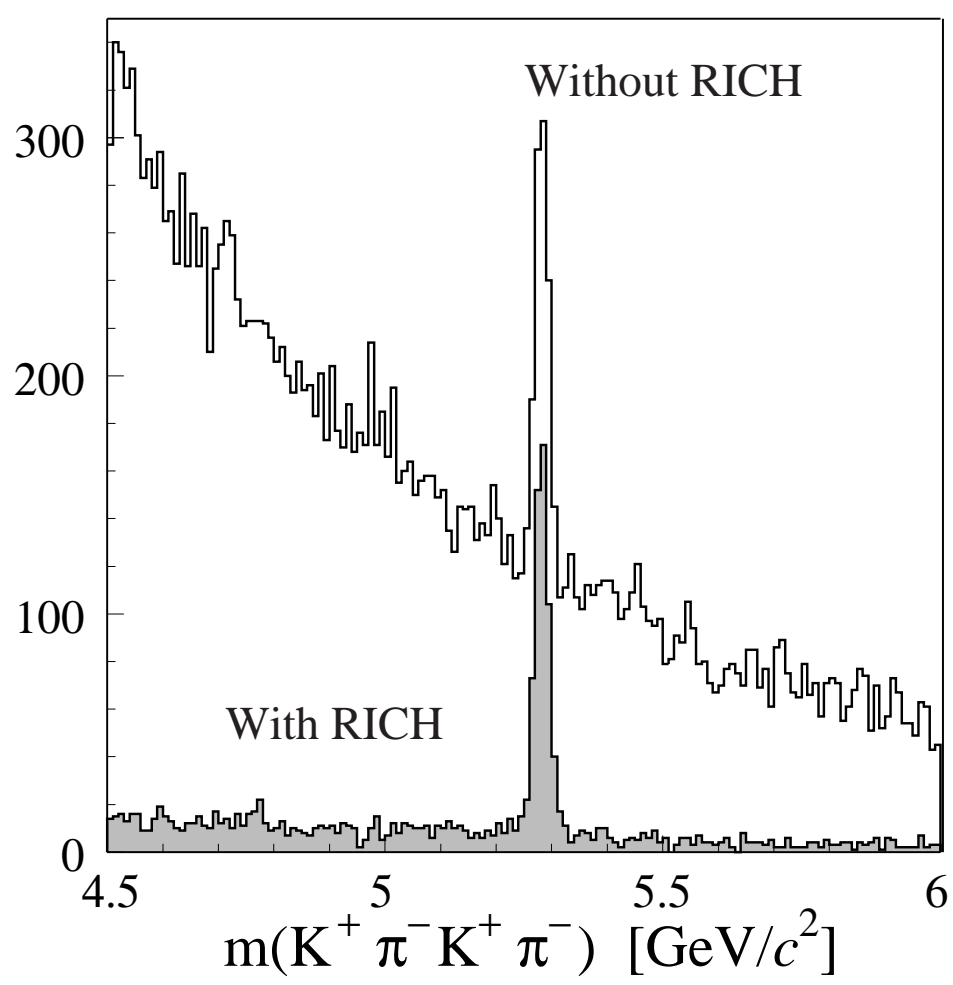

Figure 6: The $\mathrm{B}_{\mathrm{d}}^{0}$ mass peak assuming the $\mathrm{K}^{+} \pi^{-} \mathrm{K}^{+} \pi^{-}$hypothesis, with and without RICH information. 